\title{
Una revisión de la teoría de la paz democrática
}

\author{
Zeno Gobetti
}

\begin{abstract}
The idea that democracy fosters peaceful relationships among the nations is as old as at least the Kantian concept of perpetual peace, but in the twentieth century several new theories have been coined out in order to explain the behavior of democratic states towards other democracies and towards non-democratic states. These theories have been based on two interpretative models: structural/institutional and cultural/normative. This paper examines various versions of the democratic peace concept and places a particular attention at the explanatory power of the interpretative models and the role attached to the liberal values.
\end{abstract}

\section{Introducción}

La paz democrática es un clásico argumento propuesto por la tradición liberal de investigación de las relaciones internacionales. Por lo tanto, en la base de esta teoría se encuentran supuestos algunos elementos característicos de esta tradición. En particular, podemos identificar dos presupuestos fundamentales. En primer lugar, la teoría de la paz democrática se basa en la idea de que es posible limitar algunos aspectos negativos de la situación de anarquía del sistema internacional. Entre estos aspectos, la guerra es sin duda el más importante. Esto se basa en la idea de que las relaciones entre los Estados en el sistema internacional no responden necesariamente a un juego de suma cero sino que por el contrario estas relaciones pueden caracterizarse por el establecimiento de formas de cooperación y beneficio mutuo. En segundo lugar, esta teoría pierde su sentido si no se reconoce el hecho de que los Estados tienen diferentes comportamientos en el sistema internacional y que el régimen político influye de manera sustancial en tal comportamiento, aún prescindiendo de la posición de un Estado en la estructura del sistema internacional. Estas teorías, que se definen como reduccionistas, tienden a explicar el comportamiento de un Estado a partir de factores internos. Como sostiene Waltz (1979: 66): "El elemento esencial de la teoría reduccionista, por lo tanto, es que el todo se conoce a través del estudio 
de sus partes". ${ }^{1}$ En particular, esta teoría sostiene que las democracias tienden a comportarse en materia de política exterior de manera tal que puedan alcanzar las condiciones de resolución de las crisis entre los Estados sin tener que recurrir al uso de la fuerza. Esta disposición debería ser causada por algunos factores estrechamente vinculados al régimen político democrático.

Fijados estos asuntos, debemos preguntarnos por el significado del término paz democrática. Pueden presentarse por lo menos dos formas diferentes de considerar el fenómeno de la paz democrática. Un primer punto de vista se describe como "diádico" (Doyle, 1983: 205-235, 323-353) y presenta dos supuestos básicos. En primer lugar, esta versión mantiene que los Estados con un régimen democrático tienden a adoptar una conducta pacífica de relación con aquellos países con el mismo tipo de régimen. En segundo lugar, que los Estados con un sistema político democrático ejercen un comportamiento agresivo contra los Estados que tienen un régimen político no democrático. Esta hipótesis, conocida también como "paz separada" (Panebianco, 1997: 94-114), se refiere a la "comunidad de seguridad" identificada por Deutsch (1957) en la OTAN que reúne en un acuerdo de defensa militar común a los países de Europa occidental y de América del Norte (EE.UU. y Canadá). De hecho, el elemento que une a estos países es que todos ellos son democracias liberales.

Un segundo punto de vista se describe como "monádico" (Rummel, 1983: 27-71). Este sostiene una hipótesis según la cual las democracias son pacíficas en la política exterior independientemente del carácter democrático o no democrático de los otros regímenes. La hipótesis es que las democracias son pacíficas y que, como tales, entrarán en guerra contra los regímenes autoritarios sólo en caso de ser atacadas o amenazadas por ellos.

Dentro de este contexto el siguiente trabajo intentará presentar e interpretar dos versiones diferentes sobre el fenómeno de la paz democrática, que se proponen comprender cuál de estas se sostiene empíricamente sobre la base de datos estadísticos sobre la participación de las democracias en guerras. Además, presentaremos dos modelos interpretativos, "estructurales/institucionales" y "culturales/normativos", sobre la teoría recientemente mencionada.

\section{Versiones de la paz democrática}

\section{Versión monádica}

La hipótesis "monádica” de la paz democrática ha recibido particular atención tras la publicación de un artículo de Rummel en 1983 (27-71) que sintetiza

1 Traducción del autor. Waltz, K. N., Theory of international politics, McGraw-Hill, New York, 1979. 
el trabajo publicado entre 1976 y 1981 en cinco volúmenes que componen Understanding Conflict and War. En este artículo sostiene el autor dos supuestos básicos que constituyeron el fundamento de la versión monádica y que suscitaron fuertes controversias dentro de la misma tradición liberal de investigación.

La primera hipótesis enuncia que los Estados con un régimen político "libertario", tal como los define Rummel, tienden a no utilizar la violencia en las relaciones recíprocas. La segunda hipótesis, mucho más controvertida y criticada, sostiene que cuanto mayor sea el grado de "libertarismo" de un régimen político, menor será el nivel de violencia expresada por este hacia su interior y en relación con otros Estados (independientemente del tipo de régimen que caracterice a los Estados con los que entra en contacto). Sustancialmente, Rummel sostiene una versión completamente pacífica de las democracias liberales, que son propensas a la no violencia en las relaciones, no sólo entre sí sino también con Estados que cuentan con diferentes regímenes políticos. Las explicaciones facilitadas por este autor están comprendidas en los dos modelos de interpretación institucional y estructural y el modelo cultural/regulador.

En particular, una explicación presentada por Rummel hace hincapié en la importancia de la estructura económica de un régimen político. Para un "libertario", la opinión pública y los grupos de interés suponen una fuerte resistencia a la posibilidad de ir a la guerra y así evitar los costes que implica en términos de aumento de la presión fiscal y de reclutamiento para el servicio militar. Los líderes democráticos, debiendo tener en seria consideración las opiniones del electorado, tienden a adoptar una solución pacífica para evitar la escalada de la violencia que conduce al conflicto armado. Por el contrario, en un gobierno autoritario o totalitario el poder se concentra en pocas manos que, mediante el control de todos los recursos coercitivos y medios de comunicación, manipula la opinión pública y somete a los grupos de interés. Es por ello que las democracias liberales son arrastradas a la guerra contra regímenes no democráticos como consecuencia de la agresión de estos últimos.

Lo que nos interesa es hacer hincapié en que en las explicaciones dadas se presenta una mezcla de tipologías diferentes y divergentes. El período tomado en consideración por Rummel se extiende desde 1976 a 1980, utilizando la unidad de medida conocida como "dyad-years", equivalente al número de conflictos por par de países para el número de años que dura el conflicto. En total Rummel ha teorizado 62.040 dyad-years. En principio, este test hubiera puesto de manifiesto que un alto nivel de "libertarismo" está negativamente correlacionado con el grado de violencia expresado en las relaciones entre los dos Estados. Esto generó un debate sobre la paz democrática, recibiendo muchas críticas. La primera de ellas relacionada con la eficacia de las pruebas presentadas por Rummel. 
Se cuestiona, en primer lugar, la breve duración del periodo. De hecho, los años transcurridos entre 1976 y 1980 son sólo cinco, y algunos factores ligados a situaciones históricas particulares pueden influir en el resultado del análisis. Por esa misma razón, Weede (1992: 649-664) amplió el período de análisis a veinte años, entre 1960 y 1980. Esta investigación puso de manifiesto una diferencia significativa entre la participación de las democracias y las no democracias en conflictos armados, mostrando la ausencia de una significativa diferencia entre el involucramiento en guerras por parte de los regímenes democráticos en relación con las no democracias.

Paralelamente, de acuerdo con las investigaciones realizadas por Chan (1984: 617-648), la evidencia empírica cae en la dirección opuesta a las tesis Rummel. De hecho, la presencia de un régimen político "libertario" parece estar asociada con un mayor número de guerras. Por lo tanto, la idea de que las democracias están involucradas en las guerras en menor medida que otros regímenes políticos ha sido rechazada por una serie de investigaciones sucesivas. De hecho Levy (1988: 653-677), que ya había considerado como ley empírica de las relaciones internacionales la hipótesis de que las democracias no se hacían la guerra unas contra otras, admitió que éstas han participado en la guerra tanto como los regímenes no democráticos. A la misma conclusión llegaron una serie de investigaciones posteriores que consiguieron un consenso, con excepción de Rummel, respecto de tal afirmación (Gleditsch, 1992: 369-376; Starr, 1992: 41-59). Sin embargo, es necesario tener en cuenta que la investigación de Rummel se concentró en la relación entre el "libertarismo" y la violencia expresada por el Estado, y no sobre el vínculo entre "libertarismo" y la frecuencia de la participación en la guerra. En efecto, como señala Ray (1995), el mismo Rummel en el quinto volumen de Understanding Conflict and War reconoce que no se verificó una correlación entre regímenes "libertarios" y la frecuencia con la que los países que se caracterizan por este régimen resultan implicados en guerras. Su idea es que los regímenes "libertarios" resultan menos violentos en la conducción de la guerra y no más pacíficos en cuanto tales. Sin embargo, investigaciones sucesivas criticaron ambas hipótesis, argumentando que las democracias son menos propensas a la violencia y a la participación en guerras que las no-democracias, pero no en las relaciones entre ellas, y no necesariamente en las guerras en que se ven involucradas. De hecho, según Geller (1985), la frecuencia con que el Estado se ve implicado en la guerra depende de las limitaciones estructurales internas que caracterizan al régimen político. La eficacia de dichos límites se relaciona con la estabilidad interna del régimen, de manera que en presencia de un sistema político inestable, aun siendo democrático, los límites no serán capaces de desempeñar su función. 
Esto explica por qué las democracias se involucran menos en las guerras que los otros regímenes, pero no por esto deberían ser pacíficas entre sí.

Algunas investigaciones han demostrado cómo las democracias inestables tienen un nivel de conflictividad mayor al de los regímenes no democráticos. Esto se debe a que los procesos de democratización determinan un aumento de la conflictividad en el Estado en el que se desarrollan (Mansfield y Snyder, 2002: 297-338). Según Bueno de Mesquita y Lalman (1992), las democracias, por razones relacionadas con la estructura interna del sistema político, tienden a promover soluciones diplomáticas a través de negociaciones en lugar de utilizar la fuerza. Esto implica que la tendencia hacia las negociaciones diplomáticas de las democracias en un conflicto con otro Estado es independiente del hecho de que estas sean o no democracias.

De hecho, de acuerdo con Bremer (1992: 309-341), la presencia de una democracia en una crisis con otro Estado reduce la probabilidad de la guerra, generando muchas dudas sobre la idea de que las democracias se involucran en la guerra contra regímenes no democráticos en respuesta a una agresión de estos últimos. Hay casos en los que parece muy difícil establecer con precisión qué Estado es el verdadero agresor y cuál la víctima de un ataque. Ya sea porque el proceso de escalada que conduce a un conflicto militar es lento e implica una serie de pequeños enfrentamientos que degeneran en guerra o porque un Estado puede hacer uso de la guerra de manera preventiva (Gleditsch y Hegre, 1997: 283-310). En cualquier caso, la hipótesis de Rummel sobre la naturaleza no violenta de las democracias parece ser muy cuestionable por su simplismo y determinismo para describir un fenómeno complejo como la conducta de la política exterior de un Estado (MacMillan, 2003: 233-243).

Las investigaciones realizadas durante los años noventa han asociado cada vez más la versión monádica a una interpretación estructural/institucional de la paz democrática. Esto se debe a que examinando únicamente los elementos del funcionamiento interno de las democracias liberales se simplifica la teoría de la paz democrática. Sin embargo, esta simplificación ha llegado a excluir o trivializar los elementos externos que influyen en la conducta de la política exterior de una democracia. Por estas razones, la versión monádica, que puso en relieve el funcionamiento de algunos mecanismos internos para las democracias, no parece verificarse empíricamente. En contraposición, la versión diádica que ha sostenido la interpretación de la paz democrática como paz separada parece ser más útil para proporcionar una interpretación del comportamiento de política exterior de una democracia liberal. 


\section{Versión diádica}

La versión diádica de la paz democrática ha encontrado apoyo en la investigación de Doyle (1983: 205-235, 323-353). En particular en dos conocidos artículos publicados también en 1983, el autor, inspirado su análisis en la teoría kantiana de la paz perpetua ha apoyado la hipótesis de que los Estados liberales no son pacíficos como tales, sino, que sólo lo son entre ellos, mientras que resultan agresivos contra los Estados no-liberales. De hecho, la idea de que las "repúblicas" establezcan progresivamente relaciones pacíficas entre sí-al punto de unirse a través de un foedus pacificum en una federación de repúblicas y que se mantengan en un estado de guerra con Estados que no son parte de esta federación- es sin duda el núcleo de la versión diádica de la paz democrática. Aún si la explicación dada por Kant a este fenómeno es contradictoria -ya que si la opinión pública rechaza la guerra para evitar tener que sufrir los costes que ella conlleva (efecto kantiano), este rechazo incluiría a las no democracias- esta evolución desplazaría a Kant hacia una versión monádica. En cualquier caso, incluso si el origen del pensamiento de Kant de la versión diádica es dudosa, la tesis de Doyle encontró un fuerte consenso en la academia formando una nueva corriente de investigación dentro de la tradición de investigación liberal definida como "neo-kantiana" (Panebianco, 1997).

En particular, Doyle mantiene tres hipótesis que han constituido el centro del pensamiento neo-kantiano y que han caracterizado la versión diádica de la paz democrática. La primera sostiene que las democracias liberales tienden a no hacer la guerra entre ellas, la segunda que tienden aliarse entre sí y, por último, la tercera afirma que las democracias liberales son agresivas contra las no-democracias. Incluso en este caso las explicaciones dadas por Doyle mezclan elementos del modelo estructural/institucional y el modelo normativo/cultural. Sin embargo, este último pone mucha atención en el papel desempeñado por el liberalismo como elemento cultural en condiciones de establecer el efecto de la ausencia de guerra entre las democracias liberales.

Una serie de investigaciones empíricas han tratado de verificar la versión diádica de la paz democrática. En particular Maoz y Abdolali (1989: 3-35) han examinado el impacto de la guerra entre todos los posibles pares de Estados desde 1816 a 1976. Se han detectado 332 pares involucrados en guerras entre sí durante este período. En el 20\% de los casos una democracia es el agresor y en el 23\% es la agredida. Teóricamente sólo quince guerras se hubieran desarrollado entre dos democracias, por lo tanto, en proporción alrededor del 5\% de los conflictos entre dos Estados estaría compuesto por dos democracias. Sin embargo, la investigación empírica ha encontrado que un $0 \%$ de las guerras se 
da entre democracias. Esta diferencia es significativa y demostraría el efecto de la paz democrática.

Sin embargo, es importante tener en cuenta que el número de las democracias durante el período comprendido entre 1816 a 1976 ha variado de manera significativa. Su número durante el siglo XIX era muy escaso, y esto, según muchos críticos, podría explicar los resultados obtenidos por esta investigación. Por esta razón Maoz y Abdolali decidieron adoptar una medida que tuviera en consideración este factor, calculando el número de guerras entre pares de Estados por años (dyad years) observada de 1816 a 1976 para un total de 271.904 dyad years. De ellos, sólo 24.489, es decir el 9\%, involucraron la participación de democracias. Sin embargo, los datos fueron puestos en duda, sobre todo porque muchas de las guerras entre los 332 pares de Estados incluye a guerras multilaterales como si fueran separadas. Las observaciones formuladas entre un Estado contra todos los demás no pueden considerarse como independientes entre sí. ¿Cómo no pueden considerarse independientes entre sí las relaciones entre los dos Estados en los últimos años? Esto ha causado un número demasiado elevado de observaciones, alternado así el efecto de la investigación (Ray, 1995).

Maoz y Russett (1993) han proporcionado datos más fiables restringiendo el período considerado en el análisis a la segunda post-guerra, más precisamente entre 1946 y 1986. Existen tres razones por las que debe tenerse en cuenta este período para verificar empíricamente la ausencia de conflictos entre las democracias. En primer lugar, después de la Segunda Guerra Mundial el número de democracias ha aumentado considerablemente, triplicándose el número de pares de Estados democráticos en comparación con el período anterior. En segundo lugar, la posguerra se caracterizó por una serie de nuevos factores, como el desarrollo económico y los sistemas de formación de alianzas defensivas contra la amenaza de la Unión Soviética. Por último, las democracias han demostrado en este período mayor estabilidad y durabilidad, fomentando relaciones recíprocas de confianza.

Durante el período mencionado, el número de pares de Estados por año, se ha incrementado de 50 a 160, ampliando el número de casos adoptados para el examen a 265.000. Sin embargo, la mayoría de estos pares son sólo teóricos, ya que en muchos de estos no es plausible decir que hayan existido entre ellos disputas militares o diplomáticas. Además, para aclarar la investigación, es necesario restringir el análisis mediante la introducción de otros dos factores. El primero es la "contigüidad", es decir, la proximidad geográfica entre dos Estados. Uno podría esperar que la distancia reduzca la posibilidad de un conflicto, por lo que las controversias entre ellos deben ser eliminadas del cálculo. Sin embargo, es necesario examinar por separado el conflicto entre las grandes potencias, aun 
cuando no estén cerca una de otra, puesto que su escala internacional les brinda la oportunidad de realmente entrar en conflicto con otros países, aunque sean distantes. El segundo factor que limita el ámbito de la investigación es el hecho de que una de las dos partes sea una gran potencia (major power) por lo que es útil considerar como no plausibles los conflictos entre las grandes potencias y los pequeños Estados y con las colonias.

Estos límites restringen el análisis a 36.162 dyad years, con alrededor de 714 disputas entre ellos. De estas controversias, quince involucran a democracias, y sólo uno de estos conflictos se produjo entre democracias, poniendo así de manifiesto la evidencia empírica del fenómeno de la paz democrática tal como lo ha interpretado la teoría diádica. Igualmente, continúa habiendo una serie de casos ambiguos ${ }^{2}$ en los que no parece fácil diferenciar con precisión el tipo de régimen político que caracteriza a uno de los dos Estados. Definida la evidencia empírica de este fenómeno es necesario tomar en consideración las posibles explicaciones.

\section{Modelos de interpretación}

A partir de los estudios de Russett y Maoz (1993: 624-638), la investigación sobre las posibles causas de la paz democrática se ha caracterizado por los modelos interpretativos. Por supuesto, esta distinción entre explicaciones "estructurales/institucionales" y "culturales/normativas" no está exenta de críticas. No siempre es posible distinguir cuáles son fácilmente elementos estructurales y culturales, y esto crea superposiciones entre los dos supuestos que hacen la interpretación del fenómeno más confusa. Sin embargo, esta distinción continúa siendo la más utilizada por los estudiosos, principalmente por haber logrado alimentar un debate fructífero sobre la relación entre la paz y la democracia, y entre la paz y el liberalismo.

La capacidad predictiva de estas explicaciones sobre la paz o la guerra no presenta casos críticos, en tanto que tiende a predecir el mismo resultado, y presenta casos críticos cuando la previsión es mixta. Una comprobación de los méritos de una interpretación debe necesariamente tener en cuenta los casos en que la predicción empírica de los dos modelos es contrastante. Esto sirve para poner de relieve cuál de los dos presenta actualmente la mayor capacidad para interpretar el fenómeno de la paz democrática. En los párrafos siguientes se

2 Los casos dudosos en los cuales puede ser refutada la teoría de la paz democrática son: 1812 Gran BretañaEstados Unidos; 1849 Republica Romana-Francia; 1861 Guerra civil americana; 1863 Ecuador- Colombia; 1870 Francia-Prusia; 1899 Guerra de los Boer; 1898 España-Estados Unidos; 1899 Segunda guerra de las Filipinas; 1914 Primera guerra mundial, Alemania contra las democracias occidentales; 1939 Segunda Guerra Mundial, Alemania contra las democracias aliadas; 1948 Líbano-Israel; 1948 India-Pakistan; 1967 Líbano-Israel; 1974 Turquía-Chipre. 
presentarán las hipótesis planteadas por los modelos institucional/estructural y normativo/cultural, tal como fueron listados por Russett (1993). Estos serán analizados a la luz de las diferentes versiones de la paz democrática, utilizando la más reciente investigación sobre esta cuestión.

\section{Modelo institucional / estructural}

Las explicaciones llamadas "institucionales" o "estructurales" se caracterizan por la atención a factores internos del régimen político democrático. En particular, estas explicaciones se concentran en los efectos que las instituciones de la democracia ejercen sobre el comportamiento de la política exterior de un Estado. La principal tesis es que los conflictos violentos entre las democracias son raros cosa que se demuestra a través de una serie de supuestos.

1) En una democracia el equilibrio de poderes (checks and balances), la división de poderes y la presencia de un debate público en la formulación de políticas públicas, implica que la decisión de entrar en un conflicto a gran escala sea extremadamente difícil de tomar (Russett, 1993: 40).

Esta primera hipótesis es el fundamento mismo de la concepción estructural. La idea básica es que la presencia de una serie de limitaciones institucionales hace "difícil" la decisión de ir a la guerra a los líderes de gobierno. Pero, ¿qué se entiende por "difícil"? La dificultad de una decisión política puede verse de dos maneras interrelacionadas. En primer lugar, una decisión política puede ser considerada difícil si se trata de una intrincada negociación entre los actores de un sistema político. En segundo lugar, la dificultad de una decisión puede expresarse en términos del tiempo necesario para su aplicación efectiva. En este contexto, los límites estructurales que hacen difícil la decisión de la guerra en los regímenes democráticos son principalmente tres.

a) El primer elemento que haría difícil la decisión de guerra o de paz, es el equilibrio de poderes. En un sistema político caracterizado por la presencia de una serie de checks and balances, que lleva a una condición en la cual ninguno de los poderes del Estado es capaz de subyugar completamente a los otros, una decisión tan importante y arriesgada como la guerra no puede ser tomada prescindiendo del consenso de los demás poderes. Ciertamente el principio de equilibrio de poder se traduce en una estructura institucional. En particular, se concretiza en la presencia de una institución que juega el rol de balancer ${ }^{3}$ en la

3 El balancer es un poder que en un sistema político tiene el rol de mantener el equilibrio del sistema desplazando su peso político. 
estructura del sistema político. Sin embargo, la presencia de esta institución no es en sí suficiente para garantizar un equilibrio entre los poderes. De hecho, a fin de que el balancer sea capaz de desempeñar su papel, es necesario que el principio del equilibrio de poderes se considere un valor en sí mismo por todas las partes interesadas. Esto significa que este elemento esté más vinculado a una explicación normativa/cultural que a una estructural/institucional. De hecho, el concepto de equilibrio de poderes no pertenece a la tradición democrática, sino a la de pensamiento liberal.

b) El segundo elemento está estrechamente vinculado al primero. En efecto, el principio de separación de poderes no es más que una codificación jurídica del principio de equilibrio. Sin embargo, estos dos elementos no deben considerarse sinónimos. Mientras que el equilibrio de poderes aparece como un factor cultural, la división de poderes es, sin duda, un factor estructural de un régimen político. El obstáculo para la decisión de la guerra está representado por la presencia de veto players institucionales en el régimen político capaces de bloquear una decisión del gobierno (Tsebelis, 2002). El hecho de que los poderes del Estado estén separados no significa que también estén en equilibrio. Los poderes, aun separados, pueden convenir entre sí dando lugar a un sistema corporativista.

Según este punto de vista es suficiente que los poderes del Estado estén jurídicamente separados para determinar un nivel de complejidad que impida una decisión de guerra. Debemos tener en cuenta el hecho de que la decisión de ir a la guerra es tomada por un gobierno que busca la legitimidad en una votación del legislativo. Normalmente, los legislativos no niegan su consentimiento al gobierno y se limitan a ratificar la declaración de guerra. Sin embargo, el proceso de toma de decisiones debe tener en cuenta el tipo de relación institucional entre el gobierno y el legislativo. En una forma presidencial de gobierno en la que el congreso mantiene una fuerte autonomía respecto del presidente, sobre todo en una situación de gobierno dividido, ${ }^{4}$ se puede esperar que una decisión tan importante como es la guerra sea más compleja y requiera más tiempo para tomarse. En las formas parlamentarias de gobierno la negociación se lleva a cabo dentro del poder ejecutivo y el parlamento se limita a la ratificación sin generar mayores inconvenientes. De hecho, en los Estados federales, debido a la representación de los intereses locales en las instituciones centrales, el gobierno debe buscar el consentimiento de cada una de las unidades que componen la federación, lo cual hace más compleja la toma de decisiones (Silverstone, 2005: 1-55). En cualquier caso, la dificultad está representada sólo por el tiempo ne-

4 Por "gobierno dividido" se entiende una situación en la cual una de las dos cámaras, o ambas, presentan mayorías de una parte política diferente a la del presidente. 
cesario para aplicar la decisión. Por lo tanto, puede decirse que las democracias requieren más tiempo en la decisión de ir a la guerra.

c) El tercer elemento es el debate público, o la capacidad de presión de la opinión pública sobre el gobierno en un régimen político. Como hemos visto, esta idea ha sido planteada por Kant en La paz perpetua. En este punto es necesario profundizar la hipótesis tratando de entender si es cierto que el público es capaz de influir en la política exterior de un Estado y si es cierto que esta presión siempre tiende a favorecer una conducta pacífica. En lo que respecta al primer punto, hay que decir que esta presión sólo puede existir en un contexto democrático. Como Kant ha intuido, sólo en un régimen político en el que el consenso popular es necesario para acceder al gobierno, los dirigentes políticos están atentos a las demandas de la opinión pública. Si los dirigentes de una democracia decidieran ir a la guerra en contra de los deseos del público, deberían tener en cuenta los audience costs.

Como lo demuestra Gaubatz (1999), las democracias tienden a no ir a la guerra cuando se avecinan ciclos electorales para evitar una potencal pérdida de consenso y correr el riesgo de una derrota electoral. Esta idea entró en conflicto con una serie de estudios sobre el comportamiento de los presidentes de los Estados Unidos en materia de política exterior durante las elecciones (Russett, 1990). De hecho, la decisión de utilizar la fuerza puede generar, en algunos contextos, un aumento del consenso de la opinión pública. Este efecto, conocido como rally' round the flag (Lee 1977: 252-256), permitiría que los presidentes en condiciones electorales inciertas puedan obtener un aumento de la popularidad de dos a tres puntos porcentuales (Lian y Oneal, 1993: 277-300).

La teoría de la desviación, o distracción, ya sugerida por Maquiavelo y Bodino, presenta una serie de condiciones que limitan el campo de acción. La decisión del gobierno de usar la fuerza en período electoral siempre es arriesgada y, a menos de que se disponga de una serie de condiciones necesarias, la estrategia de desviación no es aplicada. En primer lugar, debe haber una certeza de la victoria con pocas bajas y, a continuación, que exista un alto diferencial de potencia contra el oponente. En cualquier caso, es dudoso el efecto sobre el consenso electoral de una victoria militar, como lo demuestran los casos de Winston Churchill y George H. Bush (Andreatta, 2005: 213-233). Por otra parte, el enemigo debe ser creíble, de lo contrario el público y los medios de comunicación no apoyarán el uso de la fuerza (Lian y Oneal, 1993: 277-300). En particular, la investigación de Geva y Mintz (1993: 484-503) ha puesto de manifiesto que las democracias tienden a no utilizar la teoría de la desviación contra otras democracias. En segundo lugar, el efecto rally' round the flag sólo funciona si el país tiene una fuerte cohesión interna. De hecho, si existen fuer- 
tes divisiones causadas por los contrastes de las minorías étnicas o religiosas, la decisión de los conflictos podrían ser perturbadora y socavar el orden interno. Por estas razones, la teoría de la desviación no está en condiciones de refutar la tesis de Gaubatz, como máximo ésta representa una rara excepción a la teoría de los ciclos electorales.

El consenso de la opinión pública en la decisión de la guerra es esencial en una democracia. La definición del interés nacional se convierte en un factor central en la obtención de consenso público (Reiter y Stam, 2002). Además, las democracias no son todas iguales. La presión de la opinión pública puede tener efectos diferentes dependiendo del tipo de estructura institucional que presenta un Estado. Es posible que haya democracias que están más abiertas que otras a la influencia de la opinión pública en materia de política exterior. Esto depende del nivel de concentración de poder que un sistema institucional presenta. En particular, la presión de la opinión pública parece ser más eficaz en las democracias en las que el poder está fragmentado y descentralizado, con instituciones débiles y con fuertes grupos sociales organizados.

Hay democracias que ofrecen una mezcla entre dos extremos, donde la presión de la opinión pública es filtrada por los partidos políticos (Risse-Kappen, 1983: 479-512). En lo que respecta al segundo punto, es necesario preguntarse por qué la opinión pública debería ser pacífica. Se pueden identificar dos tipos de explicaciones. La primera sostiene que el público siempre se opone a la guerra porque no quiere sufrir los costos, tanto económicos como en vidas humanas que ella conlleva. En cuanto a los costos económicos, la hipótesis sostiene que una intervención militar determina la necesidad de una mayor presión fiscal y esta produciría oposición. Sin embargo, no es seguro que los costos económicos de la guerra sean elevados. Por ejemplo, en el caso de un conflicto asimétrico, entre una gran potencia y un Estado pequeño, los costes económicos de la guerra serán limitados y la opinión pública podría apoyar la decisión de la guerra esperando mayores beneficios que costos (Schweller, 1992: 240-242). Esto significa que el cálculo de los costes económicos no es siempre un elemento de disuasión de los conflictos y, de hecho, en algunos casos puede incluso fomentarlos. Este punto de vista asume que el público es un actor racional capaz de evaluar con precisión el coste económico de un conflicto. La investigación sobre el papel de la opinión pública ha demostrado que las preferencias de política exterior están organizadas en una serie de "esquemas cognitivos" sobre la base de una estructura de valores más que de cálculos económicos (Hurwitz y Peffley, 1987: 1099-1120).

Por el contrario, el costo en términos de vidas humanas es siempre el elemento que más afecta la sensibilidad de la opinión pública. Sin embargo, cabe 
señalar que las "nuevas guerras" se libran principalmente por ejércitos profesionales y no por ciudadanos obligados al servicio militar (Kaldor, 1999). Por otra parte, la tecnología militar continúa reduciendo la necesidad de un gran número de hombres en la guerra, disminuyendo el riesgo y el número de pérdidas. Esto implica que incluso este tipo de costo, en parte, ha reducido su capacidad de movilizar la oposición pública a la guerra. Una segunda hipótesis sostiene que la opinión pública en una democracia es pacífica por motivos relacionados con los valores culturales que caracterizan a este régimen político. Esta segunda hipótesis hace clara referencia a un modelo normativo/cultural y, por lo tanto, se debatirá en el próximo párrafo. Lo que nos interesa en esta parte es refutar la idea de que las opiniones públicas son necesariamente pacíficas.

2) Los líderes de otros países democráticos conocen las dificultades y las limitaciones impuestas a las democracias en la decisión de ir a la guerra (Russett, 1993: 40).

Como hemos visto, las dificultades impuestas a las democracias desde el punto de vista estructural se traducen en una mayor lentitud de las mismas en la decisión de la guerra. La segunda opción presentada por este modelo sostiene que otros Estados, caracterizados por un régimen político democrático, son conscientes de estas dificultades. La idea es que las democracias son sistemas políticos "transparentes" en los que el proceso de toma de decisiones, debido a la presencia de la oposición política y la libertad de información, es completamente visible desde el exterior. Esta condición de reciprocidad de la transparencia sería capaz de crear un clima de confianza entre las democracias y de resolver el dilema de la seguridad (Doyle, 1986: 1151-1169). El reconocimiento mutuo de las intenciones pacíficas es un elemento esencial para crear ese clima de confianza entre los Estados que resulta útil para la solución pacífica de las crisis.

La transparencia reduce los efectos de los problemas de comunicación y coordinación entre los Estados democráticos (Fearon, 1994: 577-592). De hecho, en una disputa entre Estados la falta de información precisa conduce a una negociación diplomática sobre la base de bluffs recíprocos sobre la intención del uso de la fuerza. Esta diplomacia coercitiva aumenta el riesgo de una escalada militar en las crisis. Sin embargo, en las democracias la presencia de la oposición política interna favorece la transparencia de los objetivos y las modalidades para resolver las crisis internacionales por parte del gobierno. Esto explicaría la razón por la cual las democracias serían capaces de evitar que las crisis internacionales que las afectan mutuamente puedan degenerar hasta el punto de llegar a un conflicto violento (Schultz, 2001). 
Además, la defección de los acuerdos diplomáticos determina un costo interno y externo que los líderes democráticos deben tener en cuenta. En particular, la violación de los acuerdos determina los daños en términos de reputación y credibilidad de la conducta de un Estado en política exterior. Estos costos generan un incentivo para la cooperación entre las democracias (Crawford, 1994: 345-386). De acuerdo con las teorías de Bueno de Mesquita y Lalman (1992), la paz democrática puede explicarse por referencia al tipo de "imagen" que los Estados proporcionan a los demás en el sistema internacional. Si los dirigentes de un país no se ven obligados por factores de política interna a proporcionar una solución pacífica, tienden a crearse una imagen agresiva (Estados halcones) que alimentan el dilema de la seguridad. Por el contrario, los dirigentes de un Estado fuertemente limitado por la oposición política interior tienden a presentar una solución pacífica (Estados paloma). Esto hace que sea menos probable que las crisis entre las democracias den lugar a conflictos violentos.

Sin embargo, no todos los estudios están de acuerdo sobre la naturaleza transparente de la conducta de la política exterior de las democracias. Algunas investigaciones han demostrado cómo en muchos temas, especialmente relacionados con la seguridad militar, incluso las democracias tienden a mantener la mayor reserva posible (Rosato, 2003: 585-602; Finel y Lord, 1999: 325-339). Sin embargo, en las crisis entre las democracias, esta falta de información mutua no parece representar un problema como para poner en peligro la negociación diplomática. Esto se debe a la presencia de las organizaciones que desempeñan la función de gestoras de las relaciones entre los Estados democráticos (Dembinski, 2004: 545-565). Estas organizaciones desempeñan dos funciones importantes. En primer lugar, reducir la competencia por el poder entre los Estados que forman parte de ellas. Esto se demuestra por el hecho de que las alianzas entre las democracias son las más estables y duraderas entre las alianzas del sistema internacional (Gaubatz, 1996: 109-140). En segundo lugar, estas organizaciones son capaces de estabilizar la cooperación entre las democracias, desempeñando un control del cumplimiento de los acuerdos y como fuente independiente de información para la resolución de las crisis (Keohane, 1989).

Por lo tanto, la transparencia de las democracias es sin duda un factor muy importante para explicar el fenómeno de la paz democrática. Sin embargo, la explicación basada en la transparencia de información pone de relieve sólo un aspecto de ese lazo de confianza. Además, como ya hemos visto, la transparencia informativa entre las democracias puede explicarse a través del rol de las instituciones interdemocráticas más que por el efecto de factores institucionales internos de cada Estado. Por lo tanto, tenemos que comprender cuáles son los factores que llevan a cada uno de los Estados a unirse a estas instituciones. 
3) Los dirigentes de las democracias son conscientes de que otras democracias requieren tiempo para decidir y que no pueden atacar por sorpresa (Russett, 1993: 40).

Esta hipótesis se basa en la idea de que las democracias, requiriendo tiempo para decidir, no están en condiciones de organizar ataques sorpresivos contra sus adversarios. Esto implica que en una crisis entre dos democracias ambas sabrán que las negociaciones diplomáticas tendrán el tiempo necesario para completarse, sin el riesgo de ser interrumpida por un ataque por sorpresa. Este fenómeno ha sido analizado por la investigación de Schweller sobre las teorías de la evolución del sistema internacional de Gilpin (1981). Según este último, el equilibrio del sistema internacional puede cambiar como resultado del crecimiento diferencial de poder entre los Estados que lo componen. Esta situación representa un contraste entre la potencia hegemónica en declive y un creciente poder de desafío. El poder hegemónico puede decidir eliminar el oponente a través de un ataque sorpresa previendo una probable guerra de hegemonía.

El uso de la guerra preventiva se inscribe en la teoría de Gilpin como una herramienta que el poder hegemónico utiliza para mantener el status quo. Sin embargo, el mismo Gilpin subraya el hecho de que no todos los cambios en el sistema internacional se han caracterizado por las guerras preventivas y guerras de hegemonía. Existe la posibilidad de un cambio pacífico del sistema internacional. El ejemplo más evidente es el paso del papel de potencia hegemónica de Gran Bretaña a los Estados Unidos durante el siglo XX. Este paso se llevó a cabo sin que los dos países entraran en guerra el uno con el otro, lo cual se debe a que ambos presentan una serie de valores comunes. El hecho de compartir estos valores es una condición necesaria para que podamos tener un cambio pacífico del sistema internacional.

A partir de estas teorías, Schweller (1992: 240-242) fue capaz de formular una hipótesis sobre el comportamiento de las democracias en relación con la decisión sobre las guerras preventivas. Según su investigación, cuando una transición de poder involucra a una democracia en decadencia no habrá bajo ninguna circunstancia guerras preventivas. Si la potencia desafiante fuera una democracia, se llegará a un compromiso. Por el contrario, si la potencia desafiante fuera una no-democracia, el poder hegemónico democrático tenderá a crear alianzas defensivas para protegerse de ella. Sin embargo, las explicaciones en apoyo de esta teoría no están vinculadas a la lentitud de la toma de decisiones de las democracias, sino a elementos que, como veremos, se consideran parte del modelo cultural/normativo. 
Por esta razón no parece existir un vínculo directo entre la lentitud de la toma de decisiones y la ausencia de guerras preventivas. En efecto, una democracia también puede utilizar el tiempo de las negociaciones diplomáticas para prepararse contra un ataque por sorpresa por parte del enemigo. Por lo tanto, no es en este fenómeno donde se puede identificar una explicación de la ausencia de guerras preventivas entre las democracias. La segunda tesis afirma que los conflictos violentos entre las democracias y no-democracias y entre las no-democracias entre sí son frecuentes debido a que:

a) Los dirigentes de las no-democracias no están restringidos por los límites de la toma de decisiones y, por tanto, pueden decidir fácilmente, con rapidez y en secreto el comienzo de una guerra de agresión (Russett, 1993: 40).

Los regímenes autoritarios no presentan los factores internos que hemos identificado en las democracias. De hecho, en ellos no existe una separación legal de poderes ni funcionamiento eficaz del principio de equilibrio de poderes, y la presión de la opinión pública puede no tener efecto alguno sobre las opciones de política exterior del gobierno. Sin embargo, es útil introducir una distinción entre regímenes no democráticos. Los regímenes autoritarios pueden dividirse en tres tipos. En primer lugar hay regímenes militares autoritarios en los que las fuerzas armadas se han convertido en el grupo dominante en la sociedad y ejercen una forma de control o dirección eficaz de gobierno civil (Pasquino, 2000). En segundo lugar hay regímenes autoritarios personales, también conocidos como dictaduras personales, en las que sólo una persona se coloca en la parte superior de las instituciones más importantes del régimen. Por último hay regímenes autoritarios sobre la base del dominio de un solo partido. Estos regímenes, aun no teniendo los límites de las democracias, no presentan igual propensión a la guerra. En particular, la investigación de Pencey y Butler (2004: 565-581) mostró cómo los regímenes personales se caracterizan por una mayor propensión al conflicto en comparación con los regímenes de partido único. La demostración de esta hipótesis se refiere a la selectorate theory de Bueno de Mesquita.

Por otra parte, no todos los estudiosos están de acuerdo sobre el vínculo entre la paz y la democracia. De acuerdo con las teorías de Cohen (1994: $207-$ 223), formas de "paz separada" pueden existir incluso en ausencia de democracia. En particular, Kacowicz (1995: 265-276) identifica ocho áreas de paz desde 1815 hasta la fecha, sólo una de las cuales se caracteriza por tener instituciones democráticas. Sin embargo, él mismo nota como la zona de paz que se caracteriza por las instituciones democráticas presenta características peculiares. Así que si es cierto que los regímenes autoritarios no tienen los límites internos de 
las democracias, también es cierto que no todos tienen la misma propensión a los conflictos.

En cualquier caso, los regímenes autoritarios de cualquier tipo no presentan ninguna forma de transparencia sobre las intenciones de conducta de política exterior y, mucho menos, en el control de armamentos. De hecho, a menudo no aceptan el control de una organización supranacional o limitan la eficacia de sus investigaciones. En un régimen autoritario, las instituciones se consideran como instrumentos de coacción interna y, a menudo, participan en la violencia a través de un aparato de seguridad bien estructurado y, en general, gestionado por los militares. Además, son capaces de extraer y de movilizar rápidamente sus recursos internos hacia las zonas militares sin oposición (Lake, 1992: 2437). Esto conduce a un clima de desconfianza mutua que alimenta, sin duda, el dilema de la seguridad. Sin embargo, el proceso de toma de decisiones interno del gobierno no será igual entre los distintos regímenes autoritarios tomados en consideración. Los regímenes autoritarios militares y personales, tienen una menor necesidad de negociación interna en la decisión de la guerra en comparación con los regímenes de partido único. Esto se debe a que los segundos suelen tener divisiónes internas entre diversas corrientes, con varios liderazgos que chocan entre sí.

b) Los líderes de un Estado, ya sean democráticos o no democráticos, en confrontación con una no-democracia pondrán en marcha un ataque preventivo para evitar un ataque por sorpresa (Russett, 1993: 40).

Esta teoría aparece parcialmente confirmada y en parte refutada por las tesis de Schweller. De hecho, como hemos visto, este autor (1992: 240-242) sostiene que las democracias no llevan adelante nunca guerras preventivas, incluso cuando están en conflicto con una no-democracia. Las democracias tienden, en este caso, a crear alianzas defensivas entre sí para desalentar ataques preventivos por la falta de la no-democracia emergente. El intento de la democracia hegemónica de equilibrar la potencia del adversario mediante una guerra preventiva podría resultar un fracaso. Esto se debe a una serie de factores que la potencia hegemónica debe considerar. El primero, los mejores aliados democráticos del poder hegemónico serán otras democracias que en tiempos de paz no disponen de un gran ejército para utilizarlo en un ataque con el fin de proteger el prestigio de su aliado. Estas democracias prefieren descargar los costes de mantenimiento del status quo en el poder hegemónico. Si la democracia gobernante decide un ataque por sorpresa podría perder el apoyo de aliados. 
El segundo factor se caracteriza por la difficultad de encontrar una democracia no favorable al statu quo. Por el contrario, es más probable que las democracias busquen apoyar la revisión del sistema internacional mediante una política de bandwagoning 5 con la potencia no democrática desafiante. Por estas razones, la democracia hegemónica tiende a crear estructuras de defensa común que puedan actuar como disuasorias contra un ataque preventivo de la potencia emergente. Ya hemos visto que las alianzas entre las democracias probaron encontrarse entre las más estables entre las posibles alianzas del del sistema internacional. Esto implica que si el poder hegemónico es una democracia, tiende a crear estructuras de defensa común con otras similares. Por lo tanto, las democracias tienden a no practicar ataques preventivos contra las no-democracias.

En conclusión, el modelo estructural/institucional tiene muchas limitaciones en su explicación de la paz democrática. En primer lugar, el equilibrio de poderes no puede considerarse un factor institucional y, por lo tanto, no puede formar parte de ese modelo. Además, la división de poderes es capaz de ralentizar los tiempos de decisión política, pero no de bloquearla. Esto implica que la ausencia de guerras preventivas de las democracias no está vinculada a la lentitud en la toma de decisiones.

Con respecto a la opinión pública, esta no siempre se opone a la utilización de la fuerza y, de no manifestar su oposición a la guerra, los dirigentes democráticos no estáran preocupados por los audience costs. De existir una situación similar, también se desvanecerá el efecto de reducción de las guerras hechas por las democracias cuando se acercan elecciones. El único efecto estructural se puede identificar en la mayor o menor eficacia de la presión de la opinión pública sobre las decisiones del gobierno en política exterior. Esta presión cambia considerablemente, dependiendo de la forma de gobierno del país. Es por ello que tenemos que preguntarnos por qué y en qué contextos la opinión pública se manifiesta contra la guerra.

El argumento de que la oposición a la guerra está ligada a los costes económicos y a las vidas humanas que conlleva, no parece verificarse en todos los casos. Por ejemplo, la Segunda Guerra Mundial le costó a los Estados Unidos, tanto en términos económicos como de vidas humanas, mucho más que la guerra de Vietnam. Sin embargo, el juicio de la opinión pública y la presión sobre el gobierno en contra de estas dos guerras no eran los mismos. El problema de los costos no puede ser expuesto en términos absolutos, sino que debe ser considerado en términos relativos al objetivo que el conflicto se propone alcanzar. El aumento de los costos de la Segunda Guerra Mundial fue considerado por

5 Por bandwagoning se entiende la decision de apoyar a la potencia que se supone en expansión para favorecer el cambio del sistema internacional. 
los estadounidense "justo" ya que fue utilizado para un fin al que consideraban “justo”. Por lo tanto, la atención del análisis debe desplazarse hacia los factores que caracterizan la cultura de un país. En segundo lugar, hemos visto cómo las democracias no son regímenes "transparentes" como tales, sino que pueden resolver el problema de la falta información recíproca recurriendo a organizaciones interdemocráticas.

\section{Modelo cultural / normativo}

La hipótesis denominada "cultural" o "normativa" trata de explicar el fenómeno de la paz democrática a partir de factores atribuibles a fenómenos culturales. Este modelo se basa en dos presupuestos fundamentales.

1) En relación con otros Estados, los líderes tienden a seguir las mismas reglas para la resolución de los conflictos que caracterizan el proceso político interno.

2) Estos esperan que los líderes de otros Estados sigan, en la resolución de conflictos, las reglas que caracterizan su proceso político interno (Russett, 1993: 35).

De acuerdo con estos compromisos, los Estados están influenciados por el régimen político doméstico en su conducta de política exterior; no tanto por el funcionamiento interno de las estructuras institucionales, como por las normas que caracterizan la modalidad de resolución de controversias políticas internas. Estas normas, aplicadas a la política exterior, conducen a una tendencia de las democracias a la solución pacífica de las crisis con otros Estados. Esto puede proporcionar una explicación del fenómeno de la paz entre las democracias.

Esta hipótesis, planteada por Kant, sostiene que todo el proyecto de la paz perpetua entre los Estados sólo es posible si se produce el traslado de las normas que rigen el proceso político interno de la democracia al sistema internacional. El funcionamiento de estas normas es capaz de reducir los efectos nocivos de la situación de anarquía de este sistema. La tradición constructivista de investigación ha tratado de desarrollar una teoría que podría explicar el nacimiento y evolución de las normas de un sistema político dentro del sistema internacional. La investigación de Finnemore y Sikkink (1998: 887-917) han identificado tres fases del "ciclo vital" de las normas. La primera se caracteriza por la emergencia de estas. En esta etapa es importante examinar el papel desempeñado por aquellos individuos o grupos sociales que promueven la afirmación de una norma particular, cuyo funcionamiento estará condicionado por la interpretación y el 
punto de vista de aquellos que han favorecido su surgimiento. En esta etapa tienen un papel decisivo las organizaciones que son creadas para promover la aparición de la norma. Las organizaciones que favorecen la emergencia de éstas que regulan el funcionamiento del sistema internacional siguen siendo los Estados. Los primeros intentos para resolver la crisis a través del arbitraje y la mediación pueden rastrearse claramente entre muchos Estados democráticos, en particular entre Gran Bretaña y los Estados Unidos. Esto demuestra que el funcionamiento interno de estas organizaciones determina un efecto sobre el fenómeno de la emergencia de las normas en el sistema internacional.

La segunda fase se caracteriza por la aceptación de las normas. Para que una nueva entre en la práctica de la política exterior de los Estados, es necesario que se institucionalice en las organizaciones que componen el sistema internacional. El efecto de la aceptación del Estado está influenciado no sólo por el número de estos que deciden aplicar la norma sino, sobre todo, en función de cuáles sean los Estados que la adoptan. Es evidente que la decisión de adoptar una cierta norma de conducta de política exterior por parte de un poder hegemónico es capaz de influir en todo el sistema internacional en que se extiende su poder.

La tercera etapa se caracteriza por la internacionalización de la regla. El proceso de "socialización" es el mecanismo fundamental que explica el fenómeno de propagación en cascada de las normas. De acuerdo con esta teoría la aceptación de una norma por un grupo de Estados conduce a una tendencia por parte de otros en el sistema internacional a reconocer y prácticar esta norma. De hecho, las prácticas diplomáticas, tanto bilaterales como multilaterales, difundidas por el grupo de Estados que han aceptado esta regla, se ven reforzadas por una serie de incentivos y sanciones materiales.

El modelo cultural/normativo sostiene que el conflicto violento entre las democracias es extraño porque:

3) En una democracia los dirigentes tienden a resolver las diferencias políticas con el compromiso y la no violencia, respetando el derecho de oposición (Russett, 1993: 35).

Este caso pone de manifiesto el comportamiento de las democracias en la solución de los conflictos internos. La idea es que la democracia es un régimen en que la conducta violenta del Estado es sometida a una serie de fuertes restricciones. Todos los participantes en el proceso político en una democracia pueden esperar que las normas que rigen el régimen sean respetadas por todos.

En este punto debemos preguntarnos cuáles son las normas que las democracias han puesto en marcha para resolver los conflictos internos. Se pueden identificar algunos valores fundamentales del régimen. La democracia es un 
sistema competitivo, regulado y no violento para la solución de los conflictos entre las instituciones del Estado, en la cual las decisiones se toman a través del principio de mayoría. Estos principios prescriben una serie de comportamientos relacionados al funcionamiento del sistema político democrático, pero no prescriben ningún límite explícito a las políticas públicas que tal sistema puede desplegar. De ese modo, podemos identificar dos elementos reguladores comunes entre todas las democracias. En primer lugar, una democracia es siempre caracterizada por la presencia de una competencia regulada (bounded competition) (Dixon, 1994: 14-32) y no-violenta (Russett, 1993) en la que entran en conflicto intereses materiales y valores a fin de determinar las políticas públicas. Esta competencia se expresa a través del mecanismo de las elecciones. Sin embargo, la democracia no puede ser identificada simplemente como un sistema de elecciones intermitentes (Schmitter, 1991: 75-88). La competencia en el sistema político implica a varios actores de las élites políticas, a los grupos sociales que se organizan alrededor de intereses específicos y se desarrolla en diversas formas, entre las que las elecciones son sólo una modalidad.

Se puede decir que las democracias varían entre sí de acuerdo al tipo de normas que rigen la competencia interna. En segundo lugar, las decisiones de las instituciones democráticas se toman a través del criterio de la mayoría. Estos principios, fundamento del modelo democrático, a menudo se asocian con valores que se refieren a la tradición de pensamiento liberal. El vínculo entre el liberalismo y la democracia es, sin duda, muy cercano. Esto hace que sea difícil establecer una frontera entre los valores normativos de uno y otro. Sin embargo, es necesario tener en cuenta esta distinción con el fin de profundizar el análisis del vínculo entre la democracia y la paz. De hecho, muchos estudiosos tienden a identificar en los valores del liberalismo y no en los valores de la democracia la explicación del fenómeno de la paz entre las democracias liberales.

El núcleo central del liberalismo se identifica con un intento de frenar el poder político. Por lo tanto, los valores del liberalismo no se limitan a garantizar que la competencia política entre los actores en el sistema esté regulada y resulte no-violenta, tal como se establece en la democracia, sino que también las políticas públicas que implementa el gobierno sean limitadas en lo que respecta a los derechos individuales. Se puede afirmar que la práctica de la no-violencia de una democracia iliberal está limitada a los grupos que componen el sistema corporativo que la caracterizan. Mientras que en una democracia liberal el uso de la violencia se considera ilegítimo también contra las personas. Desde el punto de vista conceptual, el principio no violento de una democracia iliberal es parcial porque el uso de la fuerza se ve limitado por los derechos de los grupos sociales corporativos. Mientras que en una democracia liberal el respeto de los 
derechos individuales determina un principio no-violento mucho más amplio y no mediado por la pertenencia a un grupo social.

4) Las democracias siguen las mismas reglas para la resolución pacífica de los conflictos frente a otras democracias y esperan que estas hagan lo mismo (Russett, 1993: 35).

En este punto es preciso comprender si son los valores del sistema democrático o los del liberalismo los que determinan los incentivos internos y externos para la paz democrática. Por lo que respecta a los incentivos pacifistas internos de las democracias, podemos hacer referencia a lo que dijimos sobre el papel de la opinión pública y el efecto del sistema de elecciones en la conducta de la política exterior de un Estado. El incentivo interno más fuerte contra la decisión de la guerra de un gobierno se da por la oposición de la opinión pública. Hemos visto que las razones de tal oposición no pueden ser identificadas exclusivamente en la evaluación económica o en el costo humano de la guerra. Por lo tanto, es necesario analizar las razones culturales que impulsan a la opinión pública de un país a comprender por qué y cuándo se expresa en contra de la guerra.

Algunas investigaciones han demostrado que el público se relaciona con la política internacional a través de "esquemas cognitivos", en los cuales están presentes una serie de valores puestos en jerarquía entre sí (Conover y Feldman, 1984: 92-126). A través de estos core values la opinión pública interpreta la condición del sistema internacional (Hurwitz y Peffley, 1987: 1099-1120), pero es necesario comprender cuáles son estos valores fundamentales. Las razones por las cuales la opinión pública puede manifestarse en contra de una guerra se pueden reunir en dos tipos. En primer lugar, el público puede expresar su oposición a una guerra si considera que se violan los intereses nacionales. En segundo lugar, el uso de la violencia debe ser percibido como "legítimo" por parte del público. Si así no fuera, se encontraría con una fuerte oposición.

En lo que respecta al primer punto, se debe considerar qué es lo que se entiende por interés nacional. Un primer tipo de definición afirma que el interés nacional puede ser considerado "como la exigencia de seguridad propia de cada Estado en la condición de la anarquía de las relaciones internacionales [...]" (Pistone, 2004). Según esta definición, el concepto de interés nacional está vinculado únicamente al de seguridad nacional tan fuertemente como para ser considerados como sinónimos. Esta interpretación se consideró insatisfactoria y parcial según el punto de vista de George y Keohane (1980), quienes han tratado de redefinir el contenido de este concepto, proponiendo una diferente concepción de la seguridad y la introducción de elementos adicionales. En particular, estos autores han propuesto reemplazar el concepto de seguridad nacional por 
el concepto de la supervivencia física de los ciudadanos, objetivo a ser alcanzado incluso a expensas de la integridad territorial. También han sugerido que se incluyera como interés nacional el problema de la subsistencia económica de los ciudadanos y la integridad de las instituciones del Estado. Este último punto muestra cómo no es posible definir con precisión al interés nacional, prescindiendo del contexto constitucional de un Estado (Von Vorys, 1990). "El interés nacional "adecuadamente" concebido no puede estar en conflicto $[. .$.$] con los$ principios que dan forma a la comunidad política" (Parsi, 1998: 206).

El contexto constitucional en el que los intereses de los grupos sociales pueden expresarse y competir entre sí para definir el interés nacional es el democrático. Por esto, la tutela de las instituciones democráticas y la libertad debe ser reconocida como un elemento constitutivo del concepto de interés nacional. Por lo tanto, la opinión pública se opondrá o apoyará una guerra en base a la percepción de una amenaza a los valores constitutivos de la comunidad. En el caso de una democracia liberal la opinión pública tratará de mantener el equilibrio del sistema político que, como hemos visto, es el único verdadero límite al poder del Estado. La guerra es la mayor amenaza para el equilibrio de poderes, porque en los estados de excepción el poder tiende a concentrarse en manos del gobierno (Panebianco, 2004: 287). Es por ello que las opiniones públicas de las democracias liberales son tendencialmente contrarias a la guerra, a menos de que ésta sea considerada necesaria y "legítima" (MacMillan, 2004: 179-200).

El principio del uso legítimo de la fuerza es el segundo factor que puede determinar el apoyo o la oposición de la opinión pública. De acuerdo con MacMillan, los liberales apoyan o rechazan la guerra en base a la percepción de legitimidad de dicha elección. La legitimidad se determina sobre la base del respeto a las normas de conducta derivadas de los principios y valores de la tradición liberal (MacMillan, 2004: 179-200). Esto no significa que las democracias liberales no hagan nunca la guerra, pero, como sucede en su política interna, tienden a regular el uso de la fuerza para el cumplimiento de un conjunto de valores y principios que hagan referencia a la tradición liberal. Sin embargo, siempre es posible que en el gobierno de una democracia liberal haya un liderazgo no-liberal, y si éste se decidiera a hacer uso de la fuerza debería tener en cuenta los costes en términos de apoyo electoral.

De acuerdo con la teoría de Owen (1994: 87-125), la ideología liberal puede considerarse la causa de la paz democrática, mientras que las instituciones democráticas son el medio. La presencia de estos dos elementos puede dar una explicación a la paz democrática. Owen sostiene que los líderes liberales tienden a dar confianza a los Estados que ellos perciben como democracias liberales. En este sentido, la paz separada entre las democracias liberales no 
puede considerarse una ley general, ya que este efecto se produce sólo si estas son percibidas por las demás como tales. Esto mueve toda la atención sobre el papel desempeñado por la "percepción” de los dirigentes liberales, en particular, de la opinión pública.

Esta teoría ayuda a resolver el problema de algunos casos dudosos en el que dos democracias liberales han entrado en guerra entre sí. La presencia del régimen democrático y del liberalismo en una controversia entre dos Estados no es en sí misma una condición suficiente para que no haya guerra entre ellos. La percepción de ambas partes es crucial. No siempre es cierto que las democracias liberales se reconocen entre sí como tales, y si no es este el caso, es probable que la opinión pública no considere ilegal el uso de la fuerza. Es por ello que debemos tener en cuenta las teorías sobre los procesos de reconocimiento mutuo entre las democracias liberales.

Un proceso de reconocimiento mutuo implica la definición de "ellos" y "nosotros" como una entidad con características diferentes. La cuestión crucial es comprender lo que identifica al "nosotros" entre las democracias liberales. Como hemos dicho, Owen define al "nosotros" en términos de valores liberales. Para Risse-Kappen (1995: 491-517) la definición de la identidad debe tener en cuenta muchos factores, entre los cuales los más importantes son la estructura institucional del Estado, la visión de la política económica y los valores culturales de un país. El proceso de reconocimiento y la externalización de las normas se basan en una serie de mecanismos de comunicación de la intención pacífica relacionados con estos factores. Para Weart (1998) el proceso de reconocimiento depende de dos factores que operan en las repúblicas "oligárquicas" y democráticas: 6 el primero está dado por la presencia de la misma cultura política; el segundo, por la presencia de la cultura política "republicana", identificada en el principio del compromiso no-violento.

Estos supuestos han sido objeto de críticas por parte de Oren (1995: 147184) puesto que el concepto de democracia y el pensamiento liberal son ambos inestables en el tiempo. De hecho, éstos dependerán del contexto interno e internacional en que se encuentra un Estado. El proceso de reconocimiento no puede dejar de tener en cuenta las relaciones de fuerza entre los Estados en el sistema internacional y esto lo califica como un proceso político. Esta teoría tiene como supuesto que la paz democrática es equivalente al concepto de paz hegemónica. Por lo tanto, la paz democrática sería un producto del papel hegemónico de los Estados Unidos en el sistema internacional.

6 Para Weart las repúblicas oligárquicas se distinguen en base a la modalidad de relación con los grupos "enemigos" internos por parte de las élites en el poder. 
Sin embargo, esta hipótesis, ampliamente compartida por los realistas, no explica por qué dicha teoría está ampliamente difundida entre las democracias fuera de los Estados Unidos. En un intento de mostrar la dinámica de poder detrás de la teoría de la paz democrática, Oren ha descuidado el elemento relacional del proceso de reconocimiento. De acuerdo con Williams (2001: 525-553) el proceso de reconocimiento se basa en un proceso de construcción de la identidad tanto a nivel individual como a nivel social. La teoría de la paz democrática se fundaría en un proceso de construcción de la identidad que consta de tres fases. La primera se caracteriza por el reconocimiento de los sujetos como individuos con derechos e intereses. En la segunda, los actores se reconocen como similares y establecen una forma de gobierno que refleja estos valores (democracia liberal). En la tercera etapa se da el proceso de reconocimiento mutuo entre los gobiernos que se basa en una actitud de respeto entendido como una autolimitación de los intereses y de las acciones.

Este principio de la tolerancia es característico de las democracias liberales, que refuerzan su identidad liberal común en oposición a lo que consideran "fuera" de la comunidad liberal. Por lo tanto, el proceso de formación de "nosotros" y "ellos" está fuertemente caracterizado por la referencia a valores culturales y normativos. Este proceso de identificación involucra la opinión pública de las democracias liberales que tienden a considerar la guerra contra sus pares como un fracaso diplomático de sus dirigentes. Es por ello que en una democracia liberal los dirigentes deben tener en cuenta los audience costs relacionados con la decisión de ir a una guerra contra una equivalente.

1) Cuanto mayor sea la estabilidad de un régimen democrático, mayor será la influencia que las normas de la resolución pacífica de conflictos ejercerán sobre el comportamiento de la política exterior.

2) Para que se inicie un conflicto entre las democracias es necesario que al menos una de ellas sea un régimen político inestable (Russett 1993: 35).

Estas hipótesis enfatizan la importancia de la estabilidad de las democracias liberales en el fenómeno de la externalización de las normas. El concepto de estabilidad es un elemento fundamental para una democracia. Definimos la estabilidad de un régimen democrático con la primera alternancia en el gobierno. Esta concepción proporciona una herramienta útil para evaluar la estabilidad de un régimen democrático.

Las democracias han demostrado una mayor estabilidad que otros régimenes políticos (Gurr, 1974: 1484-1504). Sin embargo, las democracias liberales 
se caracterizan por la presencia de valores pertenecientes a la tradición liberal difundidos y enraizados en la cultura de un país. Evaluar con criterios objetivos el enraizamiento de valores normativos y culturales no es sencillo. Se puede suponer que un sistema político caracterizado por ciertos valores tienda a fomentar su difusión porque forma la base de la legitimidad de ese régimen. Cuanto mayor sea la difusión de valores liberales, mayor será el efecto que produzcan en el sistema político de un Estado.

Muchas investigaciones empíricas han demostrado una eficacia diversa durante tiempos diferentes del fenómeno de la paz democrática (Gowa, 1999). Por esta razón, algunos estudiosos han propuesto una interpretación dinámica de ésta. Como hemos visto, la idea kantiana de la paz perpetua se presenta como un ideal regulativo hacia el cual tender. Esto significa que los elementos de este proyecto también pueden ser identificados como principios reguladores. El fenómeno de la propagación de las libertades civiles, que constituye el elemento fundamental para la creación de "repúblicas" es visto como un proceso gradual de consolidación de las normas democráticas, en primer lugar en la opinión pública y, a partir de ella, en los líderes. En este sentido el buen funcionamiento de la paz democrática debe ser leído como un proceso dinámico en evolución (Cederman, 2001: 15-35). El pensamiento de Kant ha anticipado los principios de la moderna teoría de la evolución de Darwin (Modelski, 1990: 1-24). De acuerdo con esta idea, las organizaciones evolucionan como individuos a través de una serie de cambios en su estructura. Las democracias liberales son capaces de promover el desarrollo y el cambio de estructuras internas y sus relaciones exteriores.

Según Cederman los efectos de las democracias liberales son principalmente dos. En primer lugar, este proceso evolutivo produjo un cambio en el comportamiento de la política exterior. En segundo lugar, en el largo plazo, se ha podido observar una diferencia aun cada vez mayor entre las relaciones inter-democráticas y otros tipos de relaciones entre Estados.

La investigación estadística realizada por Cederman sobre la probabilidad de controversias por dyad-years muestra, con algunas excepciones temporales, un continuo descenso de las controversias entre democracias. Algunos teóricos realistas han propuesto interpretar el fenómeno de la paz democrática como un producto de la cohesión intra-sistémica de los bloques debido a la tensión bipolar de la Guerra Fría y a la presencia de armas nucleares (Mearsheimer, 1990: 5-56; Gowa, 1999). Sin embargo, las investigaciones empíricas muestran cómo el efecto de la paz democrática está en acción desde el final de la Primera Guerra Mundial y, por lo tanto, mucho antes de que los efectos de la Guerra Fría pudieran influir en la evaluación del fenómeno. Es decir, la paz democrática 
es un proceso histórico y dinámico, no estático como muchos teóricos realistas sostienen. El elemento que hace que este fenómeno sea dinámico está ligado a la característica evolutiva del pensamiento liberal (MacMillan, 1998). Los liberales consideran las relaciones internacionales como una evolución gradual e irregular hacia la libertad y la paz (Zacher y Matthew, 1995). Es posible entonces unir la relevancia del enfoque del fenómeno de la paz democrática a los procesos de difusión y mutación del pensamiento liberal.

Con la Primera Guerra Mundial la tradición de la investigación liberal ha llevado a una revisión de las teorías "ingenuas" que han apoyado la "gran ilusión" de la paz a través del comercio. El wilsonismo desempeñó un papel reformador en la investigación liberal al señalar a los estudiosos una serie de cuestiones, entre ellas la de la paz democrática (Mandelbaum 2002). El intento por lograr un nuevo orden en el sistema internacional que se inspirara en los principios liberales falló en Europa a causa de la crisis de 1929 y debido a la difusión de las ideologías nazi-fascistas. Sin embargo, en los Estados Unidos el wilsonismo ha tenido un legado indeleble en la conducta de la política exterior.

Según el modelo normativo-cultural la guerra entre los regímenes democráticos y regímenes no democráticos sucede a menudo puesto que:

1) Los dirigentes de régimen autocrático usan la violencia contra los opositores como método para resolver controversias en el proceso político interno.

2) Los regímenes autocráticos usan la violencia o amenazan con su uso en sus conflictos con otros Estados, y éstos esperan un comportamiento de este tipo.

3) Las normas de resolución de conflictos entre las democracias no tienen eficacia en las relaciones con las no-democracias, y esto obliga a las democracias a adoptar un comportamiento agresivo contra los no-democracias (Russett, 1993: 35).

Estas tres hipótesis están relacionadas con aquellas discutidas anteriormente. Con respecto a las dos primeras, hemos visto cómo la no-violencia es un rasgo distintivo del funcionamiento de los regímenes democráticos. Esto implica que todas las no democracias están inherentemente caracterizadas por un alto nivel de violencia interna. El uso de la violencia se considera una parte integral del funcionamiento del Estado en los regímenes autoritarios, ya sea militar, personal o de partido único. Lo demuestra el hecho de que en estos regímenes los aparatos de seguridad interior y militar son de especial importancia en la estructura del Estado. El funcionamiento de estos dispositivos es fundamental 
para el régimen autoritario, sin los cuales no sería capaz de resistir la presión de la oposición interna y de la opinión pública.

Los regímenes autoritarios basan su legitimidad y su papel en el uso de la fuerza contra los "enemigos" ya sea a nivel interno y externo. En esencia, estos regímenes prefieren "cortar las cabezas en lugar de contarlas". Los regímenes autoritarios, independientemente de su forma particular, tienen características comunes. En primer lugar, consideran las instituciones como medio de coacción interna para la movilización y la extracción de recursos con el fin de fortalecer el poder del Estado. En segundo, a fin de reforzar su legitimidad interna, fomentan un fuerte sentido de nacionalismo para crear una conformidad ideológica con el régimen y la difusión de una mentalidad de sacrificio individual para alcanzar los objetivos del Estado. En tercer lugar, los Estados autoritarios tienden no sólo a reforzar las estructuras de la seguridad interior y militar, sino también a militarizar la sociedad con el objetivo de propagar una mentalidad jerárquica y el respeto de la autoridad, lo cual es útil para controlar la sociedad (Schweller, 1992: 240-242).

Según Moore (1958), estos regímenes se caracterizan por una mentalidad de Estado fuerte y centralizado en condiciones de controlar y resistir a las presiones de los grupos sociales. Esta mentalidad es acompañada por una concepción técnica y amoral de la política que considera el poder del Estado como un bien en sí mismo que también se traslada a la política exterior. Esta es la razón por la cual las no-democracias tienden a amenazar con practicar ataques preventivos contra otros Estados.

Además, cómo hemos visto, la teoría de la distracción se aplica a las democracias, presentando una serie de condiciones necesarias cuya ausencia sería contraproducente para el consenso nacional. Sin embargo, no todas estas condiciones pueden ser válidas para un régimen no-democrático. De hecho, la creación de un enemigo externo puede ser coherente y distraer la atención de la sociedad en situaciones de crisis interna. En una democracia los medios de comunicación y la opinión pública desempeñan una forma de control sobre la "credibilidad" del uso de la fuerza por parte del gobierno contra otro Estado, mientras que en un régimen autoritario los medios de comunicación están bajo control del régimen.

En cuanto a la tercera opción, como ya hemos demostrado, la capacidad de las democracias para resolver sus conflictos depende del contenido liberal de su conducta de política exterior. Esto implica que las normas de conducta pueden tener un efecto sólo entre dos democracias liberales. Sin embargo, según las investigaciones de Muller (2004: 494-520), no todas las democracias liberales tienen la misma propensión a la guerra contra los regímenes iliberales. 
Se pueden distinguir dos tipos de democracias liberales. El primer tipo es definido como "militante", incluyendo en este término todas aquellas que consideran como antagónicas las relaciones con los regímenes iliberales. Estas se caracterizan por una mayor inversión en materia de armamentos y una mayor propensión a la intervención militar contra los regímenes iliberales. El segundo tipo incluye las democracias liberales "pacifistas", que se caracterizan por una tendencia a las negociaciones diplomáticas con los regímenes iliberales. Esta distinción está estrechamente vinculada a una contradicción interna en el pensamiento liberal. Incluso Doyle (1997: 251-258) observó que las democracias liberales vacilan entre una "vehemencia imprudente" y una "actitud sumisa" en la conducción de la política exterior. Esta tendencia puede entenderse si se tienen en cuenta los elementos que caracterizan al pensamiento liberal. De hecho, su elemento universalista e igualitario debería conducir a una democracia liberal a la misión de difundir el liberalismo en el mundo contra los regímenes no-liberales. Esta política intervencionista aumentaría las posibilidades de conflicto. Por otro lado, el elemento tolerante del pensamiento liberal llevaría a una democracia liberal a la decisión de la no intervención.

El modelo cultural/normativo presenta una serie de fortalezas que han hecho de la suya la interpretación más convincente. En primer lugar, este modelo es capaz de ofrecer una explicación de cómo las normas de conducta de política exterior de los Estados pueden paragonarse con las normas internas para la solución de conflictos. En segundo lugar, el modelo es capaz de presentar una teoría sobre la formación del concepto de interés nacional, teniendo en cuenta los valores políticos de la opinión pública que es, en modos diversos entre las democracias, capaz de ejercer su presión sobre el gobierno. Además, este modelo puede ofrecer, mediante la realización de un análisis de la percepción y el reconocimiento entre las democracias liberales, explicaciones útiles para la resolución de los casos dudosos donde los conflictos se han producido entre las democracias liberales.

Estas ventajas han estimulado la investigación sobre los factores culturales y normativos en la conducción de la política exterior de un Estado. Parece convincente identificar en el liberalismo el elemento cultural que más influye sobre el funcionamiento de los mecanismos institucionales que el modelo estructural identifica como la causa de la paz democrática. Por lo tanto, parece más útil considerar estas estructuras como los medios por los cuales se expresa la tendencia pacífica del liberalismo en relación con las otras democracias liberales. 


\section{Bibliografía}

-Andreatta, Filippo, (2005), "Democrazia e politica internazionale: pace separata e democratizzazione del sistema internazionale", en: Rivista italiana di Scienza Politica, anno XXXV, n. 2, pp. 213-233.

-Bremer, Stuart A., (1992), "Dangerous dyads: conditions affecting the likelihood of interstate war, 1816-1965", en: Journal of Conflict Resolution, Vol. 36, pp. 309-341.

-Bueno de Mesquita, Bruce y Lalman, David, (1992), War and reason, New Haven, Yale University Press.

-Cederman, Lars-Erik, (2001), "Back to Kant: reinterpreting the democratic peace as a macrohistorical learning process", en: American Political Science Review, Vol. 95, n. 1, marzo pp. 15-35.

-Chan, Steve, (1984), "Mirror, mirror on the wall... are the free countries more pacific?", en: Journal of Conflict Resolution, Vol. 28, pp.617-648.

-Cohen, Ronald, (1994), "Pacific unions: a reappraisal of the theory that «Democracies do not go to war with each other»", en: Journal of Peace Research, n. 20, pp. 207-223.

-Conover, P. y Feldman, S., (1984), "How people organize the political world: a schematic model”, en: American Journal of Political Science, Vol. 28, pp. 92-126.

-Crawford, Neta, (1994), "A security regime among democracies: cooperation among iroquois nations", en: International Organisation, n. 48, pp. 345-386.

-Dembinski, Matthias, Hasenclever, Andreas y Wagner, Wolfgang, (2004), "Towards an executive peace? The ambivalent effects of inter-democratic institutions on democracy, peace, and war", en: International Politics, Vol. 41, n. 4, pp. 545-565.

-Deutsch, Karl, (1957). (editado por) Political community and the north atlantic area: international organization in the light of historical experience, Princeton, Princeton Univeristy Press. 
-Dixon, William J., (1994), "Democracy and tha peaceful settlement of international conflict", en: The American Political Science Review, Vol. 88, n. 1, pp. 14-32.

-Doyle, Michael W., (1997), Ways of war and peace: realism, liberalism, and socialism, New York, Norton \& Co.

(1986), "Liberalism and world politics", en: The American Political Science Review, Vol. 80, n. 4, pp. 1151-1169.

(1983), "Kant, liberal legacies, and foreign affairs", en: Philosophy and Public Affairs, n. 12, pp. 205-235, pp. 323-353.

-Fearon, James D., (1994), "Domestic political audiences and escalation of international disputes", en: American Political Science Review, n. 88, pp. 577-592.

Finel, Bernard I. y Lord, Kristin M., (1999), "The surprising logic of trasparency”, en: International Studies Quarterly, Vol. 43, pp. 325-339.

-Finnemore, Martha y Sikkink, Kathryn, (1998), "International norm dynamics and political change", en: International Organization, Vol. 52, n. 4, pp. 887-917.

-Gaubatz, Kurt T., (1999), Elections and war: the electoral incentive in the democratic politics of war and peace, Stanford, Stanford University Press.

(1996), "Democratic states and commitment in international relations", en: International Organizations, Vol. 50, n. 1, pp. 109-140.

-Geller, Daniel S., (1985), Domestic factor in foreign policy, Cambridge, Schenman.

-Gilpin, Robert, (1981), War and change in the international system, Princeton, Princeton University Press.

-Gleditsch, Nils P., (1992), "Democracy and peace", en: Journal of Peace Research, Vol. 29, pp. 369-376. 
-Gleditsch, Nils P., y Hegre, Havard, (1997), "Peace and democracy: three levels of analysis", en: The Journal of Conflict Resolution, Vol. 41, n. 2, pp. 283310.

-Gowa, Joanne, (1999), Ballots and bullets: the elusive democratic peace, Princeton, Princeton University Press.

-Gurr, Ted R., (1974), "Persistence and change in political systems, 1800 1971", en: American Political Science Review, Vol. 48, pp. 1482-1504.

-Hurwitz, Jon y Peffley, Mark., (1987), "How are foreign policy attitudes structured?”, en: American Political Science Review, vol. 81, n. 4, pp.1099-1120.

-Kacowicz, Arie, (1995), "Explaining zones of peace: democracies as satisfied power?”, en: Journal of Peace Research, Vol. 32, n. 3, pp. 265-276.

-Kaldor, Mary, (1999), New and old wars: organized violence in a global era, Cambridge: Polity Press.

-Keohane, Robert O., (1980), “The theory of hegemonic stability and changes in international economic regimes, 1967-1977”, en: Holsti, Ole y Siverson, Randolph y George, Alexander L., Change in the international system, Boulder, Westview.

-Keohane, Robert O., (1989), International institutions and state power, Boulder, Westview Press.

-Lake, David A., (1992), "Powerful pacifists: democratic states and war", en: The American Political Science Review, Vol. 86, n. 1, , pp. 24-37.

-Lee, J. R., (1977), "Rallying around the flag: foreign policy events and presidential popularity”, en: Presidential Studies Quarterly, n. 7, pp. 252-256.

-Levy, Jack S., (1988), "Domestic politics and war", en: Journal of Interdisciplinary History, Vol. 18, pp. 653-677.

-Lian, Bradley y Oneal, John R., (1993), "Presidents , the use of military force, and public opinion", en: Journal of Conflict Resolution, Vol. 37, n. 2, pp. 277-300. 
-MacMillan, John, (2004), "Liberalism and the democratic peace", en: Review of International Studies, n. 30, pp. 179-200.

, (2003), "Beyond the separate democratic peace", en: Journal of Peace Research, Vol. 40, n. 2, pp. 233-243.

, (1998), On liberal peace. Democracy, war and the international order, London - New York, Tauris Academic Studies.

-Mandelbaum, Michael, (2002), The ideas that conquered the world: peace, democracy and free market in the twenty-first century, New York, Public Affairs.

-Mansfield, Edward y Snyder, Jack, (2002), "Democratic transitions, institutional strength and war", en: International Organization, Vol. 56, n. 2, pp. 297-338.

-Maoz, Zeev y Abdolali, Nasrin, (1989), "Regime types and international conflict", en: Journal of Conflict Resolution, Vol. 33, n. 1, pp. 3-35.

-Maoz, Zeev y Russett, Bruce, (1993), "Normative and structural causes of democratic peace, 1946-1986", en: The American Political Science Review, Vol. 87, n. 3, pp. 624-638.

-Mearsheimer, John J., (1990), "Back to the future: instability in europe after the cold war", en: International Security, Vol. 15, pp. 5-56.

-Mintz, Alex y Geva, Nehemia, (1993), "Why don't democracies fight each other? An experimental study", en: The Journal of Conflict Resolution, Vol. 37, n. 3, pp. 484-503.

-Modelski, George, (1990), "Is world politics evolutionary learning?", en: International Organization, Vol. 44, pp. 1-24.

-Moore, Barrington, (1958), Political power and social theory, New York, Harper and Row.

-Muller, Harald, (2004), "The antinomy of democratic peace", en: International Politics, Vol. 41 , pp. 494-520. 
-Oren, Ido, (1995), "The subjectivity of the "democratic" peace: changing u.S. Percepitions of imperial germany", en: International Security, Vol. 19, n. 2, pp. 147-184.

-Owen, John M., (1994), "How liberalism produces democratic peace”, en: International Security, Vol. 19, n. 2, pp. 87-125.

-Panebianco, Angelo, (2004), Il potere, lo stato, la libertà. La gracile costituzione della società libera, Bologna, il Mulino.

logna, il Mulino.

(1997), Guerrieri democratici: le democrazie e la politica di potenza, Bo-

-Parsi, Vittorio E., (1998), Interesse nazionale e globalizzazione. I regimi democratici nelle trasformazioni del sistema post-westfaliano, Milano: Jaca Book.

-Pasquino, Gianfranco, (2000), Corso di scienza politica, Bologna, il Mulino.

-Pencey, Mark y Butler, Christopher K., (2004), "The conflict behavior of authoritarian regimes”, en: International Politics, Vol. 41, pp. 565-581.

-Pistone, Sergio, (2004), "Interesse nazionale", en: Bobbio, Norberto y Matteucci Nicola y Pasquino Gianfranco, Il dizionario di politica, Torino: UTET.

-Ray, James L., (1995), Democracy and international conflict. An evaluation of the democratic peace proposition, Columbia, University of South Carolina Press.

-Reiter, Dan y Stam, Allan C., (2002), Democracies at war, Princeton: Princeton University Press.

-Risse-Kappen, Thomas, (1995), "Democratic peace - warlike democracies? A social constructivist interpretation of the liberal argument", en: European Journal of International Relations, Vol. 1, n. 4, pp. 491-517.

, (1991), "Public opinion, domestic structure, and foreign policy in liberal democracies", en: World Politics, n. 43, pp.479-512.

-Rosato, Sebastian, (2003), "The flaved logic of democratic peace theory", en: American Political Science Review, Vol. 97, n. 4, pp. 585-602. 
-Rummel, Rudolph J., (1983), “Libertarianism and interstate violence”, en: Journal of Conflict Resolution, n. 27, pp. 27-71.

-Russett, Bruce, (1993), Grasping the democratic peace:principles for a post-cold war world, New York, Princeton University Press. , (1990), Controlling the sword: The democratic governance of national security, Cambridge, Harvard Univerity Press.

-Schmitter, Philippe C. y Terry L., (1991), "What democracy is... and is not", en: Journal of Democracy, pp. 75-88.

-Schultz, Kenneth A., (2001), Democracy and coercive diplomacy, Cambridge, Cambridge University Press.

-Schweller, Randall L., (1992), "Domestic structure and preventive war: are democracies more pacific?”, en: World Politics, Vol. 44, n. 2, pp. 240-242.

-Silverstone, Scott A., 2005, "Federal democratic peace: domestic institution and international conflict in the early american republic", en: Security Studies, Vol. 13, n. 3, pp. 1-55.

-Starr, Harvey, (1992), "Why don't democracies fight one another evaluating the theory- findings feedback loop", en: Jerusalem Journal of International Relations, Vol. 14, n. 4, pp. 41-59.

-Tsebelis, George, (2002), Veto players: how political institutions work, Princeton, Princeton University Press.

-Von Vorys, Karl, (1990), American national interest. Virtue and power in foreign policy, New York, Preager Publishers.

-Waltz, Kenneth N., (1979), Theory of international politics, New York, McGraw-Hill.

-Weart, Spencer R., (1998), Never at war: why democracies will not fight one another, New Haven, Yale University Press. 
74

-Weede, Erich, (1992), "Democracy and war involvement", en: Journal of Conflict Resolution, Vol. 28, n. 4, pp. 649-664.

-Williams, Michael C., (2001), "The discipline of the democratic peace: kant, liberalism and the social construction of security communities", en: European Journal of International Relations, Vol. 7, n. 4, pp. 525-553.

-Zacher, Marck W. y Matthew, Richard A., (1995), "Liberal international theory: common threads, divergent strands", en: Kegley Jr. Charles W. (ed), Controversies in international relations theory: realism and the neoliberal challenge, New York, St. Martin's. 